(2)

\title{
Nonalcoholic steatohepatitis: emerging targeted therapies to optimize treatment options
}

This article was published in the following Dove Press journal:

Drug Design, Development and Therapy

20 August 2015

Number of times this article has been viewed

\author{
Sandra Milic' \\ Ivana Mikolasevic ${ }^{1,2}$ \\ Irena Krznaric-Zrnic' \\ Marija Stanic ${ }^{3}$ \\ Goran Poropat' \\ Davor Stimac' \\ Vera Vlahovic-Palcevski ${ }^{4}$ \\ Lidija Orlic ${ }^{2}$ \\ 'Department of Gastroenterology, \\ UHC Rijeka, Rijeka, Croatia; \\ ${ }^{2}$ Department of Nephrology, Dialysis \\ and Kidney Transplantation, UHC \\ Rijeka, Rijeka, Croatia; ${ }^{3}$ Department \\ of Hematology, UHC Rijeka, Rijeka, \\ Croatia; ${ }^{4}$ Department for Clinical \\ Pharmacology, University of Rijeka \\ Medical School, UHC Rijeka, Rijeka, \\ Croatia
}

\begin{abstract}
Diet and lifestyle changes have led to worldwide increases in the prevalences of obesity and metabolic syndrome, resulting in substantially greater incidence of nonalcoholic fatty liver disease (NAFLD). NAFLD is considered a hepatic manifestation of metabolic syndrome and is related to diabetes, insulin resistance, central obesity, hyperlipidemia, and hypertension. Nonalcoholic steatohepatitis (NASH) is an entity that describes liver inflammation due to NAFLD. Growing evidence suggests that NAFLD is a multisystem disease with a clinical burden that is not only confined to liver-related morbidity and mortality, but that also affects several extra-hepatic organs and regulatory pathways. Thus, NAFLD is considered an important public health issue, but there is currently no effective therapy for all NAFLD patients in the general population. Studies seeking optimal therapy for NAFLD and NASH have not yet led to development of a universal protocol for treating this growing problem. Several pharmacological agents have been studied in an effort to improve insulin resistance and the proinflammatory mediators that may be responsible for NASH progression. Cardiovascular risk factors are highly prevalent among NASH patients, and the backbone of treatment regimens for these patients still comprises general lifestyle interventions, including dietary changes and increased physical activity. Vitamin E and thiazolidinedione derivatives are currently the most evidence-based therapeutic options, but only limited clinical evidence is available regarding their long-term efficacy and safety. Vitamin D and renin-angiotensin-aldosterone system blockers are promising drugs that are currently being intensively investigated for use in NAFLD/NASH patients.
\end{abstract}

Keywords: nonalcoholic fatty liver disease, therapy, metabolic syndrome, insulin resistance

\section{Introduction}

Nonalcoholic fatty liver disease (NAFLD) is now the most common cause of chronic liver disease, which is predicted to be the most frequent indication for liver transplantation by $2030 .{ }^{1}$ Diet and lifestyle changes have led to worldwide increases in the prevalence of obesity and metabolic syndrome (MS), resulting in a substantially higher NAFLD incidence. NAFLD is defined as lipid-deposit accumulation in the hepatocytes, which is not due to excessive alcohol use. This disease is related to type 2 diabetes mellitus, insulin resistance (IR), central obesity, hyperlipidemia, and hypertension, and is considered a hepatic manifestation of MS. NAFLD encompasses a spectrum of diseases ranging from simple steatosis to nonalcoholic steatohepatitis (NASH), which in its most severe form can lead to liver fibrosis, cirrhosis, and hepatocellular carcinoma. ${ }^{1,2}$

NASH - an entity describing liver inflammation due to NAFLD - represents the most severe histologic form of NAFLD, defined by fat accumulation in the liver exceeding $5 \%$ of its weight. ${ }^{3}$ NAFLD does not always lead to inflammation. Patients who develop NASH can remain asymptomatic for years, but carry a serious risk of liver fibrosis/cirrhosis and hepatocellular carcinoma, and therefore of
Correspondence: Sandra Milic Department of Gastroenterology, UHC Rijeka, Krešimirova 42, 51000 , Rijeka, Croatia Tel +385 5। 658 I 22 Fax +38551658122 Emailsmilic05@gmail.com 
chronic liver failure. Although the pathogenesis of NAFLD development and progression towards NASH is somewhat unclear, IR is reportedly related to obesity and plays a central role in NAFLD pathogenesis. According to the literature, imbalances between pro- and antioxidant mechanisms and between pro- and anti-inflammatory cytokines are important components of NAFLD pathogenesis and disease progression toward NASH and fibrosis. Among the central inflammatory signaling pathways that drive the transition from chronic liver injury to fibrosis and hepatocarcinoma, it is thought that oxidative stress is involved in NAFLD onset and progression..$^{3-5}$

There exists growing evidence that NAFLD is a multisystem disease, with clinical consequences extending beyond those associated with liver-related morbidity and mortality, and affecting several extra-hepatic organs. Recent findings suggest that NAFLD increases the risks of type 2 diabetes mellitus, cardiovascular diseases (CVDs), and chronic kidney disease (CKD). Over the last decade, increasing attention has been focused on NAFLD-related CKD. ${ }^{1}$ Musso et al ${ }^{6}$ recently conducted a meta-analysis including 33 studies (63,902 participants), which revealed an association between NAFLD and increased CKD prevalence and incidence. Among patients with biopsy-proven NAFLD, NASH was associated with higher CKD prevalence and incidence compared to simple steatosis, and more advanced fibrosis was associated with higher CKD prevalence and incidence than lower fibrosis stages. Moreover, NAFLD severity was positively associated with CKD severity. Evidence also links NAFLD to other chronic diseases, including sleep apnea, colorectal cancers, osteoporosis, psoriasis, and various endocrinopathies. ${ }^{1}$

The clinical implication of these findings is that patients with NAFLD may benefit from more intensive surveillance and early treatment interventions to decrease their risks of CVD and kidney complications, as well as other diseases, such as colorectal cancer. ${ }^{1}$ Thus, NAFLD is increasingly considered an important public health issue. There is currently no therapy that is effective for all NAFLD patients in the general population. Studies aiming to identify optimal therapies for NAFLD and NASH have not yet developed a universal protocol that successfully treats this growing problem.

NAFLD is often associated with MS components and IR, and these underlying conditions play crucial roles in its pathogenesis. ${ }^{7}$ The most important factors in NAFLD pathogenesis are free fatty acids (FFA), tumor necrosis factor (TNF)-alpha, and adiponectin, which promote NAFLD by modulating the hepatic inflammatory response. Insulin allows FFA esterification and triglyceride fat storage in adipose tissue. Development of IR leads to inappropriate shifting of FFA to nonadipose tissue, such as the liver. IR increases the influx of FFA to the liver via decreased inhibition of lipolysis as well as increased de novo intrahepatic FFA synthesis (de novo lipogenesis). TNF-alpha is a proapoptotic cytokine that plays major roles in recruiting inflammatory cells to injured tissues and in promoting IR. NAFLD patients also show decreased levels of "protective adipokines", such as adiponectin, likely as a consequence of IR. Decreased adiponectin levels lead to fatty acid oxidation and increased fat accumulation in the liver. Increased hepatic FFA activate signals that increase TNF-alpha production in hepatocytes, which consequently inhibits hepatic adiponectin activity, promoting NAFLD and hepatic IR. ${ }^{8,9}$

Liver steatosis does not always result in hepatocellular injury, suggesting that additional insults are necessary. Five years ago, Tilg and Moschen ${ }^{10}$ proposed the "parallel hit hypothesis" in which many hits derived from the gut and visceral adipose tissue may act in parallel to produce hepatic inflammation, consequently leading to steatohepatitis. Moreover, IR is a common feature among NAFLD/ NASH patients and plays a central role in the complex interaction of genetic and environmental factors that contribute to NAFLD pathogenesis. Thus, improving insulin sensitivity would be expected to reduce NAFLD/NASH progression. $^{?}$

Additionally, the gut-liver axis has attracted much interest particularly regarding the pathogenesis of NAFLD because gut microbiota contribute to nutritional absorption and storage. The exact mechanisms by which gut microbiota contribute to NAFLD are poorly understood, although the role of gut microbiota in the development of NAFLD has been well documented in recent years. Thus, normalization of gut microbiota using probiotics or prebiotics is a promising treatment option for NAFLD and requires further investigations. ${ }^{11,12}$

It is likely that NAFLD/NASH management will require the treatment of not only liver disease itself but also of these associated metabolic abnormalities, as well as prevention of the "hits".

The present paper will discuss the full spectrum of investigated therapeutic options for NASH. Approaches to NAFLD/NASH treatment can generally be divided into lifestyle changes, pharmacological interventions, surgical interventions (bariatric surgery), and other interventions (phlebotomy). ${ }^{13}$ 


\section{Lifestyle changes}

Among NASH patients, cardiovascular risk factors are highly prevalent and the backbone of treatment options comprises general lifestyle interventions, including dietary changes and increased physical activity. ${ }^{14}$ Evidence suggests that a $5 \%-10 \%$ reduction of baseline body weight improves IR, aminotransferases levels, and histological findings. Thus, there are empirically based dietary recommendations that patients with NAFLD and NASH eat a hypoenergetic diet to promote weight loss. ${ }^{7}, 14$ In a randomized controlled trial, Promrat et a ${ }^{15}$ confirm that modest weight reduction (7\%-10\%) achieved through lifestyle intervention leads to improved liver histology in NASH patients. These findings support the effectiveness of body-weight reduction for patients with obesity-related NASH.

There is not yet sufficient evidence indicating what specific diet should be followed to achieve the best outcome. It is assumed that diets rich in whole grains tend to promote weight reduction, reduce liver fat, and protect against inflammation leading to NASH; however, this is not verified by clinically based evidence. ${ }^{16}$ Compared to current dietary advice, a Mediterranean diet appears to be a better option for NAFLD/NASH management. The Mediterranean diet is a pyramid characterized with the abundance of vegetable foods and cereals, such as green and yellow vegetables, salads, legumes, bread, pasta, fruits and nuts. Olive oil is the main source of fat and the intake of fish, poultry, dairy products, and eggs is moderate. In addition, different amounts of wine are usually consumed in moderation with meals. Animal fats used in butter, cream, and lard are not included in this diet. ${ }^{17}$ One study in an insulin-resistant population with NAFLD showed that even without weight loss, the Mediterranean diet reduces liver steatosis and improves insulin sensitivity. ${ }^{18}$ Moreover, in recent systematic reviews and meta-analyses, adhering to or adopting a Mediterranean dietary regimen presents a beneficial effect on the prevention and the resolution of MS, as well as on individual metabolic parameters. ${ }^{17,19}$ Additionally, reducing dietary fructose may be beneficial. Daily fructose ingestion is associated with reduced hepatic steatosis but increased fibrosis, and in older adults it is associated with hepatic inflammation and hepatocyte ballooning..$^{20,21}$ In a pediatric population, increased hepatocyte ballooning was seen in patients with reduced vitamin $\mathrm{C}$ consumption, while patients with definite NASH showed significantly higher fructose intake. ${ }^{22}$

Askari et $\mathrm{a}^{23}$ demonstrated that daily cinnamon intake may effectively improve NAFLD characteristics. As NASH is strongly associated with MS components and CVD risk, omega-3 fatty acids could be useful in MS-related conditions and can probably reduce the CVD risk in NASH patients. ${ }^{24}$ Malaguarnera et $\mathrm{a}^{25}$ demonstrated that some probiotics have beneficial effects as a complementary therapeutic approach in NAFLD patients. Their results showed that, compared to lifestyle modification alone, Bifidobacterium longum along with fructose oligosaccharides and lifestyle modification led to significantly greater reductions in steatosis and NASH activity index. ${ }^{25,26}$ Interestingly, the metabolic effects of coffee consumption have also attracted great interest among hepatology researchers. Some preliminary data suggest that coffee consumption is associated with NASH improvement. However, the exact mechanism of these beneficial effects is not yet understood and further investigation is needed. ${ }^{27}$

Exercise is another useful lifestyle-related intervention for NAFLD/NASH patients. Among patients with obesityrelated NAFLD, aerobic exercise sessions of 30-60 minutes performed three to four times weekly for 4-12 weeks are shown to improve fatty liver changes, even without accompanying body-weight reduction. However, studies investigating the usefulness of exercises in NAFLD patients have not used histological liver changes as an end point. ${ }^{7}$

In summary, current evidence suggests that a communitybased lifestyle-modification program including dietary changes and exercise targeting significant weight loss is effective in reducing liver fat in NAFLD/NASH patients..$^{28,29}$

\section{Pharmacological interventions}

Pharmacological interventions for NAFLD/NASH patients are targeted to treat underlying MS components (eg, obesity, diabetes, hypertension, and dyslipidemia) as well as liver dysfunction itself. ${ }^{7}$ Many clinical studies have investigated numerous drugs and supplements, but researchers have not yet identified a completely safe and effective therapy that can be recommended for NASH treatment. Individuals with NAFLD/NASH show heterogeneity of pathogenic pathways, necessitating individualized treatment according to the underlying pathogenesis. ${ }^{27}$

\section{Insulin sensitizers}

IR is a major mechanism in NAFLD development and progression; thus, research has focused on the potential therapeutic effect of insulin sensitizers on NAFLD/NASH. In particular, metformin and thiazolidinediones (TZDs) have been intensively investigated in terms of improving IR in NAFLD/NASH patients. The insulin sensitizer metformin is a biguanide medication that is commonly used to treat type 2 diabetes mellitus. Metformin improves IR through 
the reduction of hepatic gluconeogenesis, lipogenesis, and fatty acid oxidation; increasing peripheral and hepatic insulin sensitivity; decreasing intestinal glucose absorption; and lowering serum lipid concentration. Early pilot studies of metformin in NAFLD/NASH patients showed promising results in terms of improving fatty liver disease and aminotransferase levels, as well as reversing hepatomegaly and steatosis. ${ }^{9}$ However, in the largest randomized controlled trial, metformin failed as a treatment option compared to the placebo among NAFLD/NASH patients. ${ }^{30}$ Thus, metformin is not recommended as a therapy for NASH.

TZDs are insulin sensitizers that increase insulindependent glucose disposal and reduce hepatic glucose output by binding to peroxisome proliferator-activated receptor (PPAR) $-\gamma$. In one large study, pioglitazone induced acute improvement of adipose tissue IR that was associated with changes in liver histology, including fibrosis. ${ }^{31}$ Pioglitazone treatment is also associated with increased plasma levels of adiponectin, an adipokine that is often decreased in NASH patients. ${ }^{32}$ On the other hand, pioglitazone use is also associated with some side effects, including weight gain, edema, heart failure, and bone density reduction. Additionally, pioglitazone use for more than 2 years carries an increased risk of bladder cancer. ${ }^{33}$ In the United States, the Food and Drug Administration currently recommends avoidance of pioglitazone in cases of active bladder cancer, and caution regarding its use in patients with history of bladder cancer. ${ }^{9}$

Another TZD medication is rosiglitazone, which is widely used to treat patients with type 2 diabetes mellitus. Its safety in terms of CVD morbidity and mortality has been questioned. Rosiglitazone use is prohibited in Europe according to the recommendations of the Europe Medicines Agency, and its use is highly restricted in the USA. ${ }^{9}$ However, the latest data show that rosiglitazone use is not associated with significantly increased risk of myocardial infarction or of death from cardiovascular causes. ${ }^{34}$ Rosiglitazone treatment has a substantial antisteatogenic effect in the first year of treatment, but no additional benefit with longer therapy despite its maintained effect on insulin sensitivity and transaminase levels. This suggests that improving insulin sensitivity may not be sufficient for NASH therapy. ${ }^{35}$ It has also been noted that TZD therapy in the absence of lifestyle modifications is not effective. ${ }^{36}$ Some studies report that NASH often recurs after discontinuation of TZD therapy, ${ }^{37}$ suggesting the need for long-term therapy; however, long-term therapy is problematic due to the above-mentioned side effects of TZD use.

One attractive potential treatment involves the combination of TZDs with other medications that improve their efficacy or ameliorate their weight-gain side effects. In one study, 137 subjects with biopsy-proven NASH received $4 \mathrm{mg}$ rosiglitazone twice-daily; $4 \mathrm{mg}$ rosiglitazone and $500 \mathrm{mg}$ metformin twice-daily; or $4 \mathrm{mg}$ rosiglitazone twice-daily and $50 \mathrm{mg}$ losartan once-daily for 48 weeks. The primary outcome revealed that the treatment groups did not significantly differ in the investigated histologic parameters, indicating no greater benefit of the drug combinations compared to rosiglitazone alone. ${ }^{38}$ On the other hand, a recently published evidence-based clinical practice review article by an expert panel from the Chilean Gastroenterological Society and the Chilean Hepatology Association suggests that the combination of pioglitazone with vitamin $\mathrm{E}$ is a good pharmacological choice for patients with biopsy-proven $\mathrm{NASH}$, although evidence regarding the long-term safety and efficacy of this treatment is insufficient. ${ }^{39}$

\section{Antioxidants}

Many antioxidants are reported to show potential beneficial liver effects. Oxidative stress and depletion of endogenous antioxidants are key mechanisms responsible for liver damage and disease progression in NAFLD/NASH patients. One antioxidant that has been well-studied for NASH treatment is vitamin $\mathrm{E}$ ( $\alpha$-tocopherol), which is a fat-soluble free radical scavenger as well as a chain-breaking antioxidant in free radical reactions, such as lipid peroxidation. It is proposed that vitamin E acts on PPARs, apoptosis-regulating genes, and transforming growth factor beta 1 , which is believed to promote fibrosis. ${ }^{9,27}$

Data suggest that vitamin $\mathrm{E}$ is associated with decreasing aminotransferase levels and with improved liver histology in NASH patients. ${ }^{31,40}$ A large randomized controlled trial of adult NASH patients with and without diabetes found that, compared to placebo, daily oral vitamin E administration (800 IU/d) for 96 weeks significantly improved serum aminotransferase levels, hepatic steatosis, and lobular inflammation compared, but not the extent of hepatic fibrosis. ${ }^{41}$ More recently, the TONIC trial showed significant histological improvements in children with biopsy-proven NASH who took vitamin E. ${ }^{30}$ Another randomized controlled trial reported that 6 months of administration of vitamin $\mathrm{E}$ $(1,000 \mathrm{IU} / \mathrm{d})$ and vitamin $\mathrm{C}(1,000 \mathrm{mg} / \mathrm{d})$ resulted in significant improvement in hepatic fibrosis among patients with NASH. ${ }^{42}$ Based on the current literature, vitamin E treatment appears to be a good option for improving histology and aminotransferase activity among NASH patients. American guidelines for NAFLD management recommend the use of vitamin $\mathrm{E}$ as a first-line pharmacotherapy for nondiabetic patients with biopsy-proven NASH. 
However, it must be noted that serious concerns have been raised regarding the safety of long-term vitamin $\mathrm{E}$ treatment. Thus, physicians who chose to initiate vitamin $\mathrm{E}$ therapy should consider the potential risk of long-term treatment with this drug. In particular, vitamin $\mathrm{E}$ is not recommended in NASH patients with diabetes and high CVD risk. Treatment with vitamin $\mathrm{E}$ is also not recommended in NAFLD patients without liver biopsy, NASH cirrhosis, or cryptogenic cirrhosis. ${ }^{11,27,43}$ Actually, according to the American guidelines for the treatment of NAFLD, vitamin E is not recommended to treat NASH in diabetic patients, NAFLD without liver biopsy, NASH cirrhosis, or cryptogenic cirrhosis. ${ }^{13,44}$

There remains a need for further investigations with longer follow-up periods to examine vitamin E safety and efficacy, particularly with regard to important end points, such as prevention of cirrhosis and long-term survival in NASH patients. Future studies should also determine how long NASH patients should continue vitamin E therapy, and how different vitamin $\mathrm{E}$ formulations and sources may alter efficacy.

\section{Ursodeoxycholic acid}

Ursodeoxycholic acid (UDCA) is a hydrophilic bile acid with anti-inflammatory, antiapoptotic, and immunomodulatory properties that may make it useful for NASH treatment. Uncontrolled clinical studies show that UDCA has beneficial effects on NASH, but these results were not confirmed in a large controlled trial using UDCA. A pilot study conducted by Laurin et $\mathrm{a}^{45}$ shows that NASH treatment with UDCA for 12 months significantly improved alkaline phosphatase, ALT, and hepatic steatosis. Additionally, a randomized, double-blind, placebo-controlled multicenter trial was initiated to evaluate the efficacy and safety of high-dose UDCA (28-35 $\mathrm{mg} / \mathrm{kg}$ per day) in 126 patients with biopsy-proven NASH and elevated ALT levels. In this analysis, high-dose UDCA significantly improved aminotransferase levels, serum fibrosis markers, and selected metabolic parameters (markers of glycemic control and IR). ${ }^{46}$

On the other hand, a randomized, placebo-controlled study testing high-dose UDCA (23-28 mg/kg/day) did not show beneficial effects of UDCA in NASH treatment. Compared to placebo, this high-dose UDCA treatment failed to improve the overall histology in NASH patients. ${ }^{47}$ Overall, current data show no substantial benefit of UDCA in NASH treatment, and American guidelines do not recommend UDCA for the treatment of NAFLD/NASH. Additional studies of
UDCA treatment with histologic end points are warranted in this patient group. ${ }^{13}$

\section{Obeticholic acid}

Obeticholic acid is a synthetic bile acid that acts as a potent activator of the farnesoid $\mathrm{X}$ nuclear receptor, and that reduces liver fat and fibrosis in animal models of fatty liver disease. A recent multicenter, double-blind, placebo-controlled, parallel group, randomized clinical trial at US medical centers recently assessed efficacy of obeticholic acid in patients with non-cirrhotic NASH. The patients were randomly assigned 1:1 to receive orally administered treatment with obeticholic acid (25 mg daily) or placebo for 72 weeks. After a follow-up period, obeticholic acid was associated with improved histological features of NASH compared to placebo. ${ }^{48}$ Further studies of this drug's long-term benefits and safety are needed.

\section{Bicyclol}

Bicyclol is a synthetic compound that protects the liver against oxidation and lipid injuries, and that is widely used to treat chronic hepatitis B and C viral infections in the People's Republic of China ${ }^{49}$ Han et al ${ }^{50}$ recently investigated the efficacy of bicyclol among 248 enrolled patients with NAFLD and impaired glucose metabolism. After lifestyle changes and metformin treatment (500 $\mathrm{mg}$ orally three times daily), the patients were equally randomized to two 24-week treatment groups: bicyclol $25 \mathrm{mg}$ three times daily or vitamin $\mathrm{E}$ ( $\alpha$-tocopherol) $100 \mathrm{mg}$ three times daily (control). Liver histological assessments revealed that both groups showed decreased steatosis, inflammation, hepatocellular ballooning, and NAFLD activity scores (NAS) after treatment $(P<0.01)$. However, the decreases in inflammation and NAFLD activity scores were statistically greater in the bicyclol group compared to the vitamin E group $(P<0.01)$. Further randomized controlled studies are needed to assess the efficacy and safety of bicyclol as a treatment option for NAFLD/NASH patients.

\section{Lipid-lowering drugs}

NAFLD/NASH is strongly associated with obesity, dyslipidemia, and CVD risk. Two types of lipid-lowering drugs have been assessed for NASH treatment: 1) HMG-CoA reductase inhibitors (statins) that inhibit hepatic cholesterol synthesis; and 2) the Niemann-Pick C1-Like protein antagonist ezetimibe, which is a key player in cholesterol absorption from the small intestine. As these medications also have possible anti-inflammatory effects, lipid-lowering agents were investigated in patients with NAFLD/NASH. ${ }^{7}$ Nelson et $\mathrm{al}^{51}$ 
assessed the utility of simvastatin therapy in patients with biopsy-proven NASH and hyperlipidemia. Compared with placebo, the simvastatin group showed a $26 \%$ reduction in low-density lipoprotein, but there were no statistically significant improvements in serum aminotransferase levels, hepatic steatosis, necroinflammatory activity, or stage of fibrosis. More recently, a small study investigated the efficacy of rosuvastatin therapy (10 mg/day) for 1 year in patients with $\mathrm{MS}$, $\mathrm{NASH}$ on liver biopsy, and dyslipidemia (but without diabetes or arterial hypertension). Their preliminary findings suggest that rosuvastatin could ameliorate NASH within a year of treatment in patients with dyslipidemia. ${ }^{52}$ Finally, a recent randomized study by Takeshita et $\mathrm{al}^{53}$ reported that ezetimibe administration (10 mg/day for 6 months) was associated with improvement in hepatic fibrosis; however, ezetimibe therapy also led to increased hepatic long-chain fatty acids and HbA1c among NAFLD patients. However, a recent study on the use of ezetimibe in NASH reported that the use of ezetimibe did not significantly reduce liver fat in NASH. ${ }^{54}$

Statins decrease lipid levels both peripherally and viscerally, specifically in the liver. Consequently, aminotransferase levels may transiently increase when fat is removed from the liver. However, this transient elevation of liver enzymes does not progress to liver injury; therefore, it is not necessary to stop statin treatment if aminotransferase levels increase shortly after statin administration. ${ }^{9}$

Statins can improve the adverse outcomes of conditions commonly associated with NASH (such as hyperlipidemia, diabetes mellitus, and MS), potentially justifying their use in patients with NASH. ${ }^{55}$ While statin administration may help to improve metabolic status and reduce CVD risk in NAFLD/NASH patients, its use in NASH treatment has not been approved due to the lack of randomized controlled trials including histological evidence. ${ }^{9,56}$ Thus, there remains a need for investigations with larger sample sizes and histological evidence in NASH patients.

\section{Renin-angiotensin system blockers}

Angiotensin-converting enzyme inhibitors and angiotensin receptor blockers are attractive options for hypertension treatment. Substantial data show that these drugs are useful in slowing fibrosis progression in diabetic nephropathy and heart failure. The function of the renin-angiotensinaldosterone system (RAAS) in NAFLD has been the subject of considerable interest in recent years due to observations that this system may regulate fibrosis and tissue repair, and is thus a target for treatment with RAAS blockers. Current data suggest that the RAAS may be important in NAFLD pathogenesis, with angiotensin II (Ang II) playing a critical role in liver inflammation and fibrosis development. Thus, angiotensin-converting enzyme inhibitors and angiotensin receptor blockers could be a useful therapeutic option for NAFLD patients. ${ }^{57-61}$

The RAAS is classically conceived as a hormonal cascade responsible for controlling cardiovascular, renal, and adrenal functions, which regulates hydroelectrolytic balance and blood pressure through Ang II actions. However, recent data show that interaction against RAAS both reduces blood pressure and improves insulin sensitivity. RAAS suppression improves intracellular insulin signaling through activation of PPAR- $\gamma$, leading to improved control of adipose tissue proliferation and adipokine production. Moreover, RAAS suppression is associated with prevention of hepatic stellate cell activation and, consequently, prevention of hepatic inflammation and fibrogenesis. Additionally, local suppression of RAAS in the liver slows down the vicious cycle linking steatosis to necroinflammation, and prevents fibrosis. On the other hand, modulating the pancreatic effects of Ang II makes it possible to reach adequate insulin secretion and better metabolic balance. ${ }^{57-62}$ As mentioned earlier, IR is recognized as the pathophysiological hallmark of NAFLD. ${ }^{1}$

Several studies have investigated the efficacy of RAAS blockers, showing their beneficial effect in NAFLD patients. ${ }^{60,63-66}$ Considering the standard and wide use of RAAS blockers in the treatment of hypertension, a component of MS, further well-designed randomized controlled trials of RAAS blockers in NAFLD patients should be encouraged.

\section{Vitamin D}

Vitamin D has long been considered to mainly function only in bone metabolism. However, this vitamin has recently been recognized as a hormone with immunomodulatory, antiinflammatory, and antifibrotic properties. ${ }^{67,68}$ Vitamin D deficiency is defined as a serum 25 -hydroxyvitamin $\mathrm{D}(25(\mathrm{OH}) \mathrm{D})$ level of $<50 \mathrm{nmol} / \mathrm{L}(<20 \mathrm{ng} / \mathrm{mL})$, while serum $25(\mathrm{OH}) \mathrm{D}$ levels of $50-75 \mathrm{mmol} / \mathrm{L}(20-30 \mathrm{ng} / \mathrm{mL})$ are considered vitamin D insufficiency. ${ }^{69}$ Many authors hypothesize that low vitamin D levels are strongly associated with components of $\mathrm{MS}$, and it has been reported that serum 25(OH)D levels are inversely related to body mass index (BMI), body fat content, hypertension, IR, and diabetes. ${ }^{69,70}$

The liver is a central organ in vitamin D synthesis, where $25(\mathrm{OH}) \mathrm{D}$ occurs and where the majority of vitamin Dbinding protein is synthesized. Recently, it was recognized 
that vitamin D deficiency is frequently present in chronic liver disease, ${ }^{68}$ and an association between vitamin $\mathrm{D}$ levels and NAFLD has been observed in both adults and children. Targher et $\mathrm{al}^{71}$ reported that $25(\mathrm{OH}) \mathrm{D}$ levels were inversely correlated with histological features of biopsy-proven NAFLD/NASH. These findings have been confirmed by other authors. ${ }^{70,72-74}$ Moreover, vitamin D exerts antiproliferative and antifibrotic effects on fibrosis progression in NAFLD. This process is mediated by the antagonistic actions of VDRs on the SMAD transcription factor. VDRs are widely expressed in hepatic stellate cells, which are responsible for the excessive extracellular matrix deposition that leads to the destruction of normal liver architecture. ${ }^{75}$

The above observations regarding the link between vitamin D serum levels, NAFLD risk factors, and NAFLD severity suggest that vitamin D supplementation might represent a new therapeutic option in NASH management. Further well-designed studies are needed to investigate this possibility.

\section{Silybum marianum, or milk thistle}

During the last years, there has been considerable interest in the potential efficacy of silymarin and silybin, two natural products derived from the milk thistle (MT) plant Silybum marianum. Silybum marianum, or MT, is a well-researched plant in the treatment of various liver diseases. The active complex of MT is a lipophilic extract from the seeds of the plant and is composed of three isomer flavonolignans (silybin, silydianin, and silychristin) collectively known as silymarin. Silymarin acts as an antioxidant by reducing free radical production and lipid peroxidation, and has antifibrotic activity. ${ }^{76,77}$ In the study by Loguercio et $\mathrm{al}^{78}$ treatment with Realsil that comprises the silybin-phytosome complex (silybin plus phosphatidylcholine [PPC]) coformulated with vitamin $\mathrm{E}$ for 12 months was associated with improvement in liver enzymes, IR, and liver histology, without increases in body weight.

\section{PPC}

PPC is the main component of lecithin. PPC is an integral part of cell membranes, essential for their structural and functional integrity. Cell membranes act like gatekeepers, allowing nutrients into the cells but blocking damaging toxins from gaining entrance. PPC has been shown to enhance cell membrane function throughout the body. Horejsová et $\mathrm{al}^{79}$ in their study have shown that taking the supplement every day (along with low doses of fatty acids, B vitamins, and vitamin E) reduced fatty liver symptoms within 6 months in more than half of the study participants. In another study by Li et al ${ }^{80}$ PPC increased the breakdown of collagen, the connectivetissue protein that tends to accumulate in liver disease, promoting the scarring behind fibrosis and cirrhosis.

\section{Bariatric surgery}

Obesity and IR represent the main pathological path towards NAFLD and NASH, and thus weight loss is a first-line recommendation to individuals with NAFLD. Bariatric surgery leads to long-term weight loss and improvement in obesityrelated disorders. Caiazzo et $\mathrm{al}^{81}$ performed a prospective longitudinal study that followed 1,236 obese patients for up to 5 years after Roux-en-Y gastric bypass or adjustable gastric banding to investigate the efficacy of these surgical methods for treatment of obesity-related NAFLD. Liver biopsy samples were available for 1,201 patients at baseline, 578 patients at 1 year, and 413 patients at 5 years. Their analysis revealed that all NAFLD parameters improved after surgery, with significantly greater improvement after Rouxen-Y gastric bypass than after adjustable gastric banding.

The current evidence suggests that bariatric surgery in NAFLD/NASH patients will decrease the grade of steatosis, hepatic inflammation, and fibrosis. However, NAFLD per se is not an indication for bariatric surgery. Further investigations are needed to determine the beneficial effects of bariatric surgery in NAFLD patients who are at high risk of developing liver cirrhosis. There is also a need for more data regarding the role of bariatric surgery in modulation of NAFLD-related complications, such as CVD, diabetes, and hepatocellular carcinoma, as well as information about performing bariatric surgery before liver transplantation in cases of NASH-related liver cirrhosis. ${ }^{82-84}$

\section{Phlebotomy}

Patients with NAFLD and MS often show increased hepatic iron deposition and elevated serum ferritin levels. Available data indicate that $20 \%-30 \%$ of patients with NAFLD show hyperferritinemia with mild hepatic iron accumulation, which is commonly referred to as dysmetabolic iron overload syndrome. Besides directly inducing liver damage, excess iron is involved in MS pathogenesis by inducing adipose tissue IR and modifying adipokine release. Hyperferritinemia and increased iron stores have also been associated with hepatocellular carcinoma in patients with NAFLD. ${ }^{85,86}$

Valenti et $\mathrm{al}^{85}$ investigated phlebotomy efficacy in 38 NAFLD patients who were randomized to phlebotomy $(n=21)$ or lifestyle changes alone $(n=17)$. Phlebotomy was performed by removing $350 \mathrm{~mL}$ of blood every $10-15$ days, 
and this procedure led to no adverse events. The main study outcome was improvement of liver damage in 2 years according to NAFLD activity score. The secondary outcome was improvement in liver enzymes, including ALT, aspartate aminotransferase, and gamma-glutamyltransferase. Phlebotomy was associated with normalization of iron parameters and amelioration of liver enzymes. Compared to lifestyle changes alone, iron depletion by phlebotomy was associated with a higher rate of improvement of histological liver damage in patients with NAFLD and hyperferritinemia.

A more recent prospective 6-month randomized controlled trial examined the impact of phlebotomy on the background of lifestyle advice in NAFLD patients. Primary end points were hepatic steatosis (quantified by magnetic resonance imaging) and liver injury (determined by alanine aminotransaminase and cytokeratin-18). Secondary end points included IR measured by the insulin sensitivity index and homeostasis model of assessment, and systemic lipid peroxidation determined by plasma F2-isoprostane levels. In contrast to earlier findings, the results of this study showed that ferritin reduction by phlebotomy did not improve liver enzymes, hepatic fat, or IR among subjects with NAFLD ${ }^{87}$ To resolve this controversy, larger trials should be conducted to evaluate phlebotomy treatment in patients with severe NAFLD/NASH and hyperferritinemia resistant to lifestyle changes.

\section{Algorithm for practicing physicians on the management of NAFLD/ NASH patients}

NAFLD and NASH lack definitive etiology, effective therapy, and evidence-based clinical guidelines. NAFLD/NASH is a diagnosis of exclusion, and none of the available noninvasive diagnostic methods/tests rule out other possible underlying diseases or stage the disease for prognostic purposes. Thus, liver biopsy will often be required to confirm the diagnosis, stage the disease, rule out other liver diseases, and determine the need and urgency of aggressive therapy. ${ }^{88}$

According to the World Gastroenterology Organization recommendations for NAFLD/NASH, it is likely that NASH will be found in patients who present with risk factors such as hypertension, type 2 diabetes, sleep apnea, a positive family history, nonblack ethnicity, obesity, hyperlipidemia, sedentary lifestyle, and/or ultrasound changes consistent with fatty liver. In approaching patients with persistent elevation of liver enzymes, it is necessary to exclude other liver diseases and use of potentially hepatotoxic medications that can cause fatty liver. If risk factors for NAFLD/NASH are present (ie, MS components), the patients should be first treated with diet and exercise. The World Gastroenterology Organization recommendation for NAFLD/NASH in 2014 is a weight loss goal of $5 \%-10 \%$, with a $25 \%$ decrease in calories from a normal diet. Moderate exercise three to four times per week is suggested as an exercise goal, as well as the proper control of diabetes, hyperlipidemia, and other cardiovascular risks. In the absence of improvement, and with rather abnormal ALT levels after 6 months' follow-up, a liver biopsy should be considered. ${ }^{88}$

In the presence of potential signs of liver cirrhosis, AST $>$ ALT, and low platelet and albumin levels in initial evaluation of patients with suspected NAFLD, liver biopsy should be considered. Patients with simple steatosis have good prognosis provided they receive treatment of the cardiovascular risk factors. If the diagnosis of NASH is confirmed, the treatment depends on patient's BMI and associated risk factors. If the patient's BMI is $<35 \mathrm{~kg} / \mathrm{m}^{2}$, we advise first treatment with diet, exercise, and behavior modifications. If the patient's BMI is $>40 \mathrm{~kg} / \mathrm{m}^{2}$ or $>35 \mathrm{~kg} / \mathrm{m}^{2}$ in the presence of associated risk factors, we advise treatment with diet, exercise, and behavior modifications, and also consideration of treatment with bariatric surgery. As it was mentioned, there is no evidence-based approved drug therapy for NAFLD/NASH to date. Thus, experimental therapy (including antioxidants and antifibrotic agents, such as vitamin E and pentoxifylline) should be considered by experienced clinicians in patients who fail to achieve 5\%-10\% weight reduction in 6 months to 1 year of successful lifestyle changes. ${ }^{88}$

\section{Conclusion}

While much progress has been made in elucidating the epidemiology, natural history, and pathogenesis of NAFLD, there remains no effective therapy for NAFLD patients in the general population. Several pharmacological agents have been studied in an effort to improve IR and the proinflammatory mediators that may be responsible for NASH progression. Additional investigations are warranted to establish the efficacy and safety of these medications. It is also important to treat the metabolic abnormalities that are often associated with NAFLD/NASH, such as hypertension, diabetes, dyslipidemia, and obesity. Statin therapy should be considered for patients with NAFLD/NASH and hypercholesterolemia, while treatment with TZDs may be initiated for NAFLD/NASH patients with diabetes. RAAS blockers could be helpful for NAFLD/NASH patients with hypertension, especially if diabetes is also present. NASH patients show a high prevalence of cardiovascular risk factors, and 
the backbone of treatment regimens for these patients still constitutes general lifestyle interventions, including dietary changes and increased physical activity.

\section{Disclosure}

The authors report no conflicts of interest in this work.

\section{References}

1. Byrne CD, Targher G. NAFLD: a multisystem disease. J Hepatol. 2015; 62(1 Suppl):S47-S64.

2. Koplay M, Sivri M, Erdogan H, Nayman A. Importance of imaging and recent developments in diagnosis of nonalcoholic fatty liver disease. World J Hepatol. 2015;7:769-776.

3. Marchesini G, Bugianesi E, Forlani G, et al. Nonalcoholic fatty liver, steatohepatitis, and the metabolic syndrome. Hepatology. 2003;37:917-923.

4. Lee JJ, Lambert JE, Hovhannisyan Y, et al. Palmitoleic acid is elevated in fatty liver disease and reflects hepatic lipogenesis. Am JClin Nutr. 2015;101: 34-43.

5. Mazo DF, de Oliveira MG, Pereira IV, et al. S-nitroso-N-acetylcysteine attenuates liver fibrosis in experimental nonalcoholic steatohepatitis. Drug Des Devel Ther. 2013;7:553-563.

6. Musso G, Gambino R, Tabibian JH, et al. Association of non-alcoholic fatty liver disease with chronic kidney disease: a systematic review and meta-analysis. PLoS Med. 2014;11:e1001680

7. Watanabe S, Hashimoto E, Ikejima K, et al. Evidence-based clinical practice guidelines for nonalcoholic fatty liver disease/nonalcoholic steatohepatitis. J Gastroenterol. 2015;50:364-377.

8. Chaldakov GN, Stankulov IS, Hristova M, Ghenev PA. Adipobiology of disease: adipokines and adipokine-targeted pharmacology. Curr Pharm Des. 2003;9:1023-1031.

9. Takahashi Y, Sugimoto K, Inui H, Fukusato T. Current pharmacological therapies for nonalcoholic fatty liver disease/nonalcoholic steatohepatitis. World J Gastroenterol. 2015;21:3777-3785.

10. Tilg H, Moschen AR. Evolution of inflammation in nonalcoholic fatty liver disease: the multiple parallel hits hypothesis. Hepatology. 2010;52: 1836-1846.

11. Abenavoli L, Scarpellini E, Rouabhia S, Balsano C, Luzza F. Probiotics in non-alcoholic fatty liver disease: which and when. Ann Hepatol. 2013; $12: 357-363$.

12. Miura K, Ohnishi H. Role of gut microbiota and Toll-like receptors in nonalcoholic fatty liver disease. World J Gastroenterol. 2014;20:7381-7391.

13. Chalasani N, Younossi Z, Lavine JE, et al. The diagnosis and management of non-alcoholic fatty liver disease: practice guideline by the American Association for the Study of Liver Diseases, American College of Gastroenterology, and the American Gastroenterological Association. Hepatology. 2012;55:2005-2023.

14. Le TA, Loomba R. Management of Non-alcoholic Fatty Liver Disease and Steatohepatitis. J Clin Exp Hepatol. 2012;2:156-173.

15. Promrat K, Kleiner DE, Niemeier HM, et al. Randomized controlled trial testing the effects of weight loss on nonalcoholic steatohepatitis. Hepatology. 2010;51:121-129.

16. Ross AB, Godin JP, Minehira K, Kirwan JP. Increasing whole grain intake as part of prevention and treatment of nonalcoholic Fatty liver disease. Int J Endocrinol. 2013;2013:585876.

17. Abenavoli L, Milic N, Peta V, Alfieri F, De Lorenzo A, Bellentani S. Alimentary regimen in non-alcoholic fatty liver disease: Mediterranean diet. World J Gastroenterol. 2014;20:16831-16840.

18. Ryan MC, Itsiopoulos C, Thodis T, et al. The Mediterranean diet improves hepatic steatosis and insulin sensitivity in individuals with non-alcoholic fatty liver disease. J Hepatol. 2013;59:138-143.

19. Kastorini CM, Milionis HJ, Esposito K, Giugliano D, Goudevenos JA, Panagiotakos DB. The effect of Mediterranean diet on metabolic syndrome and its components: a meta-analysis of 50 studies and 534,906 individuals. J Am Coll Cardiol. 2011;57:1299-1313.
20. Jin R, Welsh JA, Le NA, et al. Dietary fructose reduction improves markers of cardiovascular disease risk in Hispanic-American adolescents with NAFLD. Nutrients. 2014;6:3187-3201.

21. Abdelmalek MF, Suzuki A, Guy C, et al. Increased fructose consumption is associated with fibrosis severity in patients with nonalcoholic fatty liver disease. Hepatology. 2010;51:1961-1971.

22. Vos MB, Colvin R, Belt P, et al. Correlation of vitamin E, uric acid, and diet composition with histologic features of pediatric NAFLD. J Pediatr Gastroenterol Nutr. 2012;54:90-96.

23. Askari F, Rashidkhani B, Hekmatdoost A. Cinnamon may have therapeutic benefits on lipid profile, liver enzymes, insulin resistance, and high-sensitivity C-reactive protein in nonalcoholic fatty liver disease patients. Nutr Res. 2014;34:143-148.

24. Janczyk W, Socha P, Lebensztejn D, et al. Omega-3 fatty acids for treatment of non-alcoholic fatty liver disease: design and rationale of randomized controlled trial. BMC Pediatr. 2013;13:85.

25. Malaguarnera $M$, Vacante $M$, Antic $T$, et al. Bifidobacterium longum with fructo-oligosaccharides in patients with nonalcoholic steatohepatitis. Dig Dis Sci. 2012;57:545-553.

26. Ferolla SM, Armiliato GN, Couto CA, Ferrari TC. Probiotics as a complementary therapeutic approach in nonalcoholic fatty liver disease. World J Hepatol. 2015;7:559-565.

27. Baran B, Akyüz F. Non-alcoholic fatty liver disease: what has changed in the treatment since beginning? World J Gastroenterol. 2014;20: $14219-14229$.

28. Wong VW, Chan RS, Wong GL, et al. Community-based lifestyle modification programme for non-alcoholic fatty liver disease: a randomized controlled trial. J Hepatol. 2013;59:536-542.

29. Yoshimura E, Kumahara H, Tobina $\mathrm{T}$, et al. Lifestyle intervention involving calorie restriction with or without aerobic exercise training improves liver fat in adults with visceral adiposity. J Obes. 2014;2014: 197216.

30. Lavine JE, Schwimmer JB, Van Natta ML, et al. Effect of vitamin E or metformin for treatment of nonalcoholic fatty liver disease in children and adolescents: the TONIC randomized controlled trial. JAMA. 2011;305:1659-1668.

31. Bell LN, Wang J, Muralidharan S, et al. Relationship between adipose tissue insulin resistance and liver histology in nonalcoholic steatohepatitis: a pioglitazone versus vitamin $\mathrm{E}$ versus placebo for the treatment of nondiabetic patients with nonalcoholic steatohepatitis trial follow-up study. Hepatology. 2012;56:1311-1318.

32. Gastaldelli A, Harrison S, Belfort-Aguiar R, et al. Pioglitazone in the treatment of NASH: the role of adiponectin. Aliment Pharmacol Ther. 2010; 32:769-775.

33. Lewis JD, Ferrara A, Peng T, et al. Risk of bladder cancer among diabetic patients treated with pioglitazone: interim report of a longitudinal cohort study. Diabetes Care. 2011;34:916-922.

34. Stone JC, Furuya-Kanamori L, Barendregt JJ, Doi SA. Was there really any evidence that rosiglitazone increased the risk of myocardial infarction or death from cardiovascular causes? Pharmacoepidemiol Drug Saf. 2015;24:223-227.

35. Argo CK, Northup PG, Al-Osaimi AM, Caldwell SH. Systematic review of risk factors for fibrosis progression in non-alcoholic steatohepatitis. J Hepatol. 2009;51:371-379.

36. Ratziu V, Giral P, Jacqueminet S, et al. Rosiglitazone for nonalcoholic steatohepatitis: one-year results of the randomized placebo-controlled Fatty Liver Improvement with Rosiglitazone Therapy (FLIRT) Trial. Gastroenterology. 2008;135:100-110.

37. Ratziu V, Charlotte F, Bernhardt C, et al. Long-term efficacy of rosiglitazone in nonalcoholic steatohepatitis: results of the fatty liver improvement by rosiglitazone therapy (FLIRT 2) extension trial. Hepatology. 2010;51:445-453.

38. Torres DM, Jones FJ, Shaw JC, Williams CD, Ward JA, Harrison SA. Rosiglitazone versus rosiglitazone and metformin versus rosiglitazone and losartan in the treatment of nonalcoholic steatohepatitis in humans: a 12-month randomized, prospective, open-label trial. Hepatology. 2011; $54: 1631-1639$. 
39. Arab JP, Candia R, Zapata R, et al. Management of nonalcoholic fatty liver disease: an evidence-based clinical practice review. World J Gastroenterol. 2014;20:12182-12201.

40. Hoofnagle JH, Van Natta ML, Kleiner DE, et al. Vitamin E and changes in serum alanine aminotransferase levels in patients with non-alcoholic steatohepatitis. Aliment Pharmacol Ther. 2013;38:134-143.

41. Sanyal AJ, Chalasani N, Kowdley KV, et al. Pioglitazone, vitamin E, or placebo for nonalcoholic steatohepatitis. N Engl J Med. 2010;362: 1675-1685.

42. Harrison SA, Torgerson S, Hayashi P, Ward J, Schenker S. Vitamin E and vitamin $C$ treatment improves fibrosis in patients with nonalcoholic steatohepatitis. Am J Gastroenterol. 2003;98:2485-2490.

43. Watanabe S, Hashimoto E, Ikejima K, et al. Evidence-based clinical practice guidelines for nonalcoholic fatty liver disease/nonalcoholic steatohepatitis. Hepatol Res. 2015;45:363-377.

44. Rinella ME. Nonalcoholic fatty liver disease: a systematic review. JAMA. 2015;313:2263-2273.

45. Laurin J, Lindor KD, Crippin JS, et al. Ursodeoxycholic acid or clofibrate in the treatment of non-alcohol-induced steatohepatitis: a pilot study. Hepatology. 1996;23:1464-1467.

46. Ratziu V, de Ledinghen V, Oberti F, et al. A randomized controlled trial of high-dose ursodesoxycholic acid for nonalcoholic steatohepatitis. J Hepatol. 2011;54:1011-1019.

47. Leuschner UF, Lindenthal B, Herrmann G, et al. NASH Study Group. High-dose ursodeoxycholic acid therapy for nonalcoholic steatohepatitis: a double-blind, randomized, placebo-controlled trial. Hepatology. 2010;52:472-479.

48. Neuschwander-Tetri BA, Loomba R, Sanyal AJ, et al. Farnesoid X nuclear receptor ligand obeticholic acid for non-cirrhotic, non-alcoholic steatohepatitis (FLINT): a multicenter, randomised, placebo-controlled trial. Lancet. 2015;385:956-965.

49. Liu GT. Bicyclol: a novel drug for treating chronic viral hepatitis B and C. Med Chem. 2009;5:29-43

50. Han Y, Shi JP, Ma AL, Xu Y, Ding XD, Fan JG. Randomized, vitamin Econtrolled trial of bicyclol plus metformin in non-alcoholic fatty liver disease patients with impaired fasting glucose. Clin Drug Investig. 2014; 34:1-7.

51. Nelson A, Torres DM, Morgan AE, Fincke C, Harrison SA. A pilot study using simvastatin in the treatment of nonalcoholic steatohepatitis: a randomized placebo-controlled trial. J Clin Gastroenterol. 2009; 43:990-994.

52. Kargiotis K, Katsiki N, Athyros VG, et al. Effect of rosuvastatin on nonalcoholic steatohepatitis in patients with metabolic syndrome and hypercholesterolaemia: a preliminary report. Curr Vasc Pharmacol. 2014; 12:505-511.

53. Takeshita Y, Takamura T, Honda M, et al. The effects of ezetimibe on non-alcoholic fatty liver disease and glucose metabolism: a randomized controlled trial. Diabetologia. 2014;57:878-890.

54. Loomba R, Sirlin CB, Ang B, et al. Ezetimibe for the treatment of nonalcoholic steatohepatitis: assessment by novel magnetic resonance imaging and magnetic resonance elastography in a randomized trial (MOZART trial). Hepatology. 2015;61:1239-1250.

55. Eslami L, Merat S, Malekzadeh R, Nasseri-Moghaddam S, Aramin H. Statins for non-alcoholic fatty liver disease and non-alcoholic steatohepatitis. Cochrane Database Syst Rev. 2013;12:CD008623.

56. Mansourian PG, Yoneda M, Krishna Rao M, Martinez FJ, Thomas E, Schiff ER. Effects of statins on the risk of hepatocellular carcinoma. Gastroenterol Hepatol (N Y). 2014;10:417-426.

57. Paschos P, Tziomalos K. Nonalcoholic fatty liver disease and the rennin-angiotensin system: implications for treatment. World J Hepatol. 2012;4:327-331.

58. Abbas G, Silveira MG, Lindor KD. Hepatic fibrosis and the renninangiotensin system. Am J Ther. 2011;18:e202-e208.

59. Tobli JE, Muñoz MC, Cao G, Mella J, Pereyra L, Mastai R. ACE inhibition and AT1 blockade prevent fatty liver and fibrosis in obese Zucker rats. Obesity (Silver Spring). 2008;16:770-776.
60. Orlic L, Mikolasevic I, Lukenda V, Anic K, Jelic I, Racki S. Nonalcoholic fatty liver disease and the renin-angiotensin system blockers in the patients with chronic kidney disease. Wien Klin Wochenschr. 2015; 127:355-362.

61. Orlić L, Mikolasevic I, Bagic Z, Racki S, Stimac D, Milic S. Chronic kidney disease and nonalcoholic Fatty liver disease-is there a link? Gastroenterol Res Pract. 2014;2014:847539.

62. Grace JA, Herath CB, Mak KY, Burrell LM, Angus PW. Update on new aspects of the rennin-angiotensin system in liver disease: clinical implications and new therapeutic options. Clin Sci (Lond). 2012;123:225-239.

63. Pereira RM, dos Santos RAS, da Costa Dias FL, Teixeira MM, Simões e Silva AC. Renin-angiotensin system in the pathogenesis of liver fibrosis. World J Gastroenterol. 2009;15:2579-2586.

64. Hirata T, Tomita K, Kawai T, et al. Effect of telmisartan for treatment of nonalcoholic fatty liver disease: Fatty Liver Protection Trial by telmisartan or losartan study (FANTASY). Int J Endocrinol. 2013;2013: 587140 .

65. Goh GB, Pagadala MR, Dasarathy J, et al. Renin-angiotensin system and fibrosis in non-alcoholic fatty liver disease. Liver Int. 2015;35:979-985.

66. Georgescu EF, Ionescu R, Niculescu M, Mogoanta L, Vancica L. Angiotensin-receptor blockers as therapy for mild-to-moderate hypertension-associated non-alcoholic steatohepatitis. World $J$ Gastroenterol. 2009;15:942-954.

67. Kitson MT, Roberts SK. D-livering the message: the importance of vitamin D status in chronic liver disease. J Hepatol. 2012;57:897-909.

68. Küçükazman M, Ata N, Dal K, et al. The association of vitamin D deficiency with nonalcoholic fatty liver disease. Clinics (Sao Paulo). 2014; 69:542-546

69. Han YP, Kong M, Zheng S, et al. Vitamin D in liver disease: from mechanisms to clinical trials. J Gastroenterol Hepatol. 2013;28 Suppl 1: 49-55.

70. Strange RC, Shipman KE, Ramachandran S. Metabolic syndrome: A review of the role of vitamin D in mediating susceptibility and outcome. World J Diabetes. 2015;6:896-911.

71. Targher G, Bertolini L, Scala L, et al. Association between serum 25-hydroxyvitamin D3 concentrations and liver histology in patients with non-alcoholic fatty liver disease. Nutr Metab Cardiovasc Dis. 2007; 17:517-524

72. Dasarathy J, Periyalwar P, Allampati S,et al. Hypovitaminosis D is associated with increased whole body fat mass and greater severity of non-alcoholic fatty liver disease. Liver Int. 2014;34:e118-127.

73. Manco M, Ciampalini P, Nobili V. Low levels of 25-hydroxycitamin $\mathrm{D}(3)$ in children with biopsy-proven nonalcoholic fatty liver disease. Hepatology. 2010;51:2229.

74. Nobili V, Giorgio V, Liccardo D, et al. Vitamin D levels and liver histological alterations in children with nonalcoholic fatty liver disease. Eur J Endocrinol. 2014;170:547-553.

75. Potter JJ, Liu X, Koteish A, Mezey E. 1,25-dihydroxyvitamin D3 and its nuclear receptor repress human $\alpha 1$ (I) collagen expression and type I collagen formation. Liver Int. 2013;33:677-686.

76. Abenavoli L, Capasso R, Milic N, Capasso F. Milk thistle in liver diseases: past, present, future. Phytother Res. 2010;24:1423-1432.

77. Del Ben M, Polimeni L, Baratta F, Pastori D, Loffredo L, Angelico F. Modern approach to the clinical management of non-alcoholic fatty liver disease. World J Gastroenterol. 2014;20:8341-8350.

78. Loguercio C, Andreone P, Brisc C, et al. Silybin combined with phosphatidylcholine and vitamin $\mathrm{E}$ in patients with nonalcoholic fatty liver disease: a randomized controlled trial. Free Radic Biol Med. 2012;52: 1658-1665.

79. Horejsová M, Urban J. [The effect of polyene phosphatidylcholine (Essentiale forte) in the treatment of liver steatosis and ultrasound findings preliminary study]. Cas Lek Cesk. 1994;133:366-369. Czech.

80. Li J, Kim CI, Leo MA, Mak KM, Rojkind M, Lieber CS. Polyunsaturated lecithin prevents acetaldehyde-mediated hepatic collagen accumulation by stimulating collagenase activity in cultured lipocytes. Hepatology. 1992;15:373-381. 
81. Caiazzo R, Lassailly G, Leteurtre E, et al. Roux-en-Y gastric bypass versus adjustable gastric banding to reduce nonalcoholic fatty liver disease: a 5-year controlled longitudinal study. Ann Surg. 2014;260:893-898; discussion 898-899.

82. Hafeez S, Ahmed MH. Bariatric surgery as potential treatment for nonalcoholic fatty liver disease: a future treatment by choice or by chance? J Obes. 2013;2013:839275.

83. Milić S, Lulić D, Štimac D D. Non-alcoholic fatty liver disease and obesity: biochemical, metabolic and clinical presentations. World J Gastroenterol. 2014;20:9330-9337.

84. Milić S, Stimac D. Nonalcoholic fatty liver disease/steatohepatitis: epidemiology, pathogenesis, clinical presentation and treatment. Dig Dis. 2012;30:158-162.

85. Valenti L, Fracanzani AL, Dongiovanni P, et al. A randomized trial of iron depletion in patients with nonalcoholic fatty liver disease and hyperferritinemia. World J Gastroenterol. 2014;20:3002-3010.
86. Dongiovanni P, Fracanzani AL, Fargion S, Valenti L. Iron in fatty liver and in the metabolic syndrome: a promising therapeutic target. J Hepatol. 2011;55:920-932.

87. Adams LA, Crawford DH, Stuart K, et al. The impact of phlebotomy in nonalcoholic fatty liver disease: a prospective, randomized, controlled trial. Hepatology. 2015;61:1555-1564.

88. Review Team, LaBrecque DR, Abbas Z, et al. World Gastroenterology Organisation global guidelines: Nonalcoholic fatty liver disease and nonalcoholic steatohepatitis. J Clin Gastroenterol. 2014;48:467-473.

\section{Publish your work in this journal}

Drug Design, Development and Therapy is an international, peerreviewed open-access journal that spans the spectrum of drug design and development through to clinical applications. Clinical outcomes, patient safety, and programs for the development and effective, safe, and sustained use of medicines are a feature of the journal, which has also been accepted for indexing on PubMed Central. The manuscript management system is completely online and includes a very quick and fair peer-review system, which is all easy to use. Visit http://www.dovepress.com/testimonials.php to read real quotes from published authors.

Submit your manuscript here: http://www.dovepress.com/drug-design-development-and-therapy-journal 